\title{
Emerging recognition of the complement system in hepatic ischemia/reperfusion injury, liver regeneration and recovery (Review)
}

\author{
ZHI-GAO HU ${ }^{1,2^{*}}$, YI ZHOU ${ }^{1 *}$, CHENG-JIE LIN ${ }^{1,3}$, GUAN-DOU YUAN $^{1}$ and SONG-QING HE ${ }^{1}$ \\ ${ }^{1}$ Division of Hepatobiliary Surgery, The First Affiliated Hospital of Guangxi Medical University, Nanning, \\ Guangxi 530021; ${ }^{2}$ Department of General Surgery, The First Affiliated Hospital of Nanchang University, \\ Nanchang, Jiangxi 330006; ${ }^{3}$ Department of Hepatopancreatobiliary Surgery, The First Affiliated \\ Hospital of Fujian Medical University, Fuzhou, Fujian 350005, P.R. China
}

Received November 12, 2019; Accepted November 26, 2020

DOI: $10.3892 /$ etm.2021.9654

\begin{abstract}
Hepatic ischemia/reperfusion injury (IRI) is a result of the ischemic cascade and may occur in the settings of liver trauma, resection and transplantation. Components of the complement system have been indicated to be mediators of hepatic IRI and regulators of liver regeneration. As such, their potential to mediate both beneficial and harmful effects render them key targets for therapy. In the present study, the mechanisms of complement mediating hepatic IRI were discussed with a focus on the different functions of complement in hepatic injury and liver recovery, and an explanation for this apparent paradox is provided, i.e. that the complement products $\mathrm{C} 3 \mathrm{a}$ and $\mathrm{C} 5 \mathrm{a}$ have an important role in liver damage; however, $\mathrm{C} 3 \mathrm{a}$ and $\mathrm{C} 5 \mathrm{a}$ are also necessary for liver regeneration. Furthermore, situated at the end of the complement activation cascade, the membrane attack complex is crucial in hepatic IRI and inhibiting the complex with a site-targeted murine complement inhibitor, complement receptor 2-CD59, may improve liver regeneration after partial hepatectomy, even when hepatectomy is combined with ischemia and reperfusion.
\end{abstract}

\section{Contents}

1. Introduction

Correspondence to: Professor Song-Qing He or Dr Guan-Dou Yuan, Division of Hepatobiliary Surgery, The First Affiliated Hospital of Guangxi Medical University, 6 Shuangyong Road, Nanning, Guangxi 530021, P.R. China

E-mail: dr_hesongqing@163.com

E-mail: yuan510149375@163.com

*Contributed equally

Key words: complement, complement inhibitor, liver, ischemia/ reperfusion injury, regeneration
2. Complement-mediated injury cascades in hepatic IRI

3. Complement cascades are essential in liver recovery and regeneration

4. Complement regulation between hepatic IRI and liver regeneration

5. Future perspective

\section{Introduction}

Ischemia/reperfusion injury (IRI) of the liver occurs when there is a transient blockage of blood supply to the organ, followed by re-establishment of the blood supply, which results in severe injury to the liver. Hepatic IRI is frequently associated with severe liver trauma and operations on the liver, such as hepatic lobe resection and liver transplantation $(1,2)$. IRI causes an acute inflammatory response, which may lead to local and remote liver cell damage and dysfunction $(3,4)$. Numerous crucial elements of the pathogenesis of hepatic IRI have been identified. These include activated polymorpho-nuclear leukocytes (PMNs) and Kupffer cells (KCs), formation of oxygen radicals and activation of complement (5-7). Complement activation is critical during hepatic IRI, which may rapidly promote complement activation through activating PMNs and $\mathrm{KCs}$ by the release of cellular proteins, thereby leading to the formation of reactive oxygen species (ROS) and the promotion of inflammatory response (8).

Studies in animal models of hepatic IRI have increased the understanding of the pathogenesis of the complement system in IRI and guided clinical investigations aimed at preventing or minimizing this condition. Blockade of complement activation has been determined to reduce hepatic IRI (9). Of note, in experimental models of IRI, targeted complement inhibitors complement receptor 2 (CR2)-complement receptor1-related protein (Crry) and CR2-CD59 were demonstrated to improve the ability of the liver to recover after injury $(10,11)$.

In the present review, the current knowledge of complement activation in the pathophysiology of hepatic IRI is summarized with a focus on the importance of targeted complement inhibitors in regulating both the injury and the recovery from 
IRI, highlighting relevant novel approaches and experimental discoveries that may improve the clinical management of liver transplantation and resection surgery.

\section{Complement-mediated injury cascades in hepatic IRI}

Although the exact mechanisms of hepatic IRI remain to be fully elucidated, factors involved in liver injury include sinusoidal congestion and microcirculatory dysfunction, adhesion-molecule expression, aggregation and activation of leukocyte and platelets, free radicals, pro-inflammatory cytokines and complement activation products $(12,13)$.

In general, there are two phases of hepatic injury follow IRI (14). The first phase ( $<2 \mathrm{~h}$ after reperfusion) is considered oxidative stress, which is induced by KCs. The KCs are able to produce and release a large quantity of ROS in this phase. Accumulation of ROS may induce mitochondria-dependent apoptosis and has a key role in hepatic injury (15), which was confirmed by providing partial protection against hepatic IRI with free radical scavengers, chemical antioxidants and blockage of KC activity with gadolinium chloride (16-18). The second phase is characterized by the recruitment of activated neutrophils into the liver parenchyma (19-21). The recruitmentis initiated during the first phase of hepatic IRI and may activate a series of inflammatory pathways. During the second phase of hepatic IRI, KCs and infiltrating neutrophils further increase the ROS and pro-inflammatory cytokines, leading to irreversible tissue damage in the form of sinusoidal congestion, cytoplasmic vacuolation of hepatocytes, necrosis and apoptosis $(21,22)$. Most importantly, complement products are also critically involved in $\mathrm{KC}$ activation and neutrophil infiltration (8).

In IRI, complement activation results in the rapid induction of multiple parallel downstream pathways of hepatic injury. Studies in animal models have indicated that IRI in several organ systems results in activation of complement components through eitherthe antibody-dependent classical pathway (23), the alternative pathway (24) or the mannose-binding lection-mannan-binding lectin serine protease pathway (25). Activation of complement by any of these pathways results in the formation of biologically active potent inflammatory complement substances, including the anaphylatoxins (C3a and C5a) and the cytolytic membrane attack complex (MAC) (26). Diffusible anaphylatoxin-mediated responses and MAC-mediated lysis of hepatocytes may have parallel roles in exacerbating hepatic IRI.

The anaphylatoxins C3a and C5a are both crucial chemotactic factors for leukocytes. They also contribute to the accumulation and infiltration of PMNs, induce smooth muscle contraction, increase vascular permeability, cause the release of histamine and activate KCs (27-29). Activated KCs and PMNs secrete inflammatory cytokines, such as TNF- $\alpha$ and IL-1 $\beta$ (30-32), which amplify the inflammatory response and cause hepatic injury. Furthermore, inflammatory cells may also release myeloperoxidase and toxic products when activated, resulting in the production of ROS, which may directly injure hepatocytes. In addition, endothelium activated by complement results in the production of numerous factors, such as adhesion molecules, cytokines, platelet-activating factors, leukotrienes, P-selectin and endothelin (33); activity of these factors may result in fibrin deposition, platelet aggregation and adhesion of neutrophils to the vascularendothelium (34). This complement-associated mechanism contributes to impaired hepatic microcirculatory blood flow in the reperfusion period, which has an important role in hepatic IRI (12). The anaphylatoxins $\mathrm{C} 3 \mathrm{a}$ and $\mathrm{C} 5 \mathrm{a}$ also have a significant role in renal and cerebral IRI $(35,36)$.

In the terminal complement pathway, the MAC is formed, which directly causes cell lysis and stimulates cells to release pro-inflammatory molecules, which may amplify the inflammatory response by promoting the expression of pro-inflammatory mediators (37). Furthermore, the MAC may influencethe recruitment of inflammatory cells, adhesion of leukocytes to endothelium and enhancement of the pro-coagulant properties of the endothelium (38). While the MAC promotes hepatocyte lysis, it may also be a parallel injury pathway during hepatic IRI. Direct cellular injury via this mechanism also triggers inflammation, thus amplifying the hepatic injury response. A previous study has proved that livers of C6-deficient rats (blocked in only the terminal pathway) are protected from the deleterious effects of IRI (39). It has provided direct evidence for an important role of the MAC in hepatic IRI. Therefore, inhibition of the complement cascade may be a novel approach for minimizing liver damage and preventing fatal organ failure after hepatic ischemia. Furthermore, a study by our group has indicated that steatotic livers are more sensitive to IRI and complement $\mathrm{C} 3$ has a critical role during IRI (40). On this basis, it may be suggested that complement inhibition with CR2-Crry maybe a strategy for reducing liver-donor shortage by allowing greater use of marginal steatotic donor livers (hepatic steatosis typically renders the donor organ unusable, as donor organs with $>30 \%$ steatosis are more likely to develop graft failure).

\section{Complement cascades are essential in liver recovery and regeneration}

Liver regeneration may occur after partial hepatectomy, liver transplantation, viral infection, IRI or toxic injury. The regeneration process is complex and well-orchestrated $(41,42)$, and it has been widely studied in order to understand the mechanisms that regulate hepatocyte proliferation and survival $(43,44)$. In normal adult mouse liver, mature differentiated hepatocytes are not vegetative and are quiescent. However, when the liver receives a regenerative stimulus (e.g., resection or toxic injury), $\sim 95 \%$ of hepatocytes undergo cell division and maintain their metabolic functions (45). The process of regeneration is tightly regulated through controlled delivery of cytokines, growth factors, paracrine signals and complement activation products that control the proliferation of hepatic cells (46). The initiation step is activation by priming of the quiescent hepatocytes (G0 phase) with factors such as TNF- $\alpha$, IL-6 and nitric oxide, which then provides a regenerative stimulus that promotes entry into the cell cycle (G1 phase) by complete mitogens such as hepatic growth factor (HGF), ligands for epidermal growth factor receptor (EGFR), transforming growth factor- $\alpha$, EGF, heparin-binding EGF and amphiregulin (47). Furthermore, the cytokines TNF- $\alpha$ and IL- 6 are crucial in regulating the early priming phase of liver regeneration by contributing to the mitogenic priming of hepatocytes and the early hepato- 
cyte proliferative response of the liver (48). These cytokines are components of the TNF- $\alpha / \mathrm{TNFR} 1 / \mathrm{NF}-\kappa \mathrm{B} / \mathrm{IL}-6 / \mathrm{STAT} 3$ pathway that drives hepatocyte gene expression, although the inhibitors and stop signals of hepatic regeneration remain to be elucidated (49).

Recent evidence indicates that complement activation products serve important physiological roles in liver regeneration and recovery, after either resection or toxic injury (50-53). Mice deficient in C3 and/or C5 after partial hepatectomy and toxic injury with carbon tetrachloride have an impaired regenerative response, which may be reversed by reconstitution of C3a and C5a. Furthermore, interception of C5a receptor (C5aR) signaling may suppress IL-6/TNF- $\alpha$ induction, and lack of C3aR and C5aR stimulation attenuates NF- $\kappa$ B-STAT-3 activation after hepatectomy, which abrogates the proliferative response of hepatocytes to liver injury $(50,52)$. According to one study, restoration of hepatocyte proliferative capabilities in $\mathrm{C}_{3} \mathrm{aR}^{-/-}$mice by systemic $\mathrm{C} 3 \mathrm{a}$ reconstitution was not beneficial (50). Another similar study indicated that treatment with C5a agonist in rats after partial hepatectomy facilitated the synthesis of the growth factor/receptor pair HGF/c-Met to upregulate the expression of HGF and c-Met mRNA, which significantly increased the expression of cyclin E and D1mRNA levels and incorporation of 5-bromodeoxuridin (54). Taken together, these data indicate that: i) $\mathrm{C} 3 \mathrm{a}$ and $\mathrm{C} 5 \mathrm{a}$ signaling are critical for the early priming phase of liver regeneration; ii) $\mathrm{C} 3 \mathrm{a}$ exerts its regenerative effects via the $\mathrm{C} 3 \mathrm{aR}$; and iii) $\mathrm{C} 5 \mathrm{a}$ is upregulated during liver regeneration. Binding ofC5a to the $\mathrm{C} 5 \mathrm{aR}$ promotes a growth response by inducing the expression of HGF mRNA and its corresponding c-MET receptor. Furthermore, $\mathrm{C} 5 \mathrm{aR}$ is also involved in a cell-cycle signaling pathway $(54,55)$.

With regard to complement activation pathways, a study reported that animals in which all of the traditional upstream C3 activation pathways were disrupted, i.e. C4-null mice treated with complement factor B neutralizing antibody (monoclonal antibody1379), exhibited normal C3 activation and hepatocellular proliferative response after partial hepatectomy. The study concluded that a non-traditional mechanism of complement activation was involved in the regenerative process (53).

\section{Complement regulation between hepatic IRI and liver regeneration}

After hepatic resection, the liver undergoes both IRI injury and regeneration. As outlined above, complement activation is necessary for liver regeneration and is extensively involved in hepatic IRI. Thus, it was postulated that a balance exists, in which excessive complement activation (which may occur in liver resection) or excessive inhibition or inadequate blockade of complement activity may be deterrent to regeneration. This hypothesis is supported in part by the results of a study by our group (56), in which C3deficiencyor inhibition of complement was achieved by using an inhibitor of C3 activation and CR2-Crry was indicated to protect against hepatic IRI; CR2-mediated targeting of complement inhibition has the potential to be of therapeutic benefit for numerous complement-associated diseases or disease states (57), such as traumatic brain injury, age-related macular degeneration and collagen antibody-induced arthritis (58-61). Although either C3 deficiency or high-dose CR2-Crry protected against IRI in mice, they significantly increased injury and impaired regeneration in the partial hepatectomy model (56). However, a low dose of CR2-Crry actually enhanced regeneration after partial hepatectomy (56). Furthermore, this dose was protective in a model of IRI, albeit somewhat less protective than the higher dose (56). Given these results, the effect of C3 deficiency and dose of complement inhibitor were examined in a model incorporating both IRI and 70\% partial hepatectomy (which mimics the procedure used for massive liver resection under the Pringle maneuver) (56). In the combined model, either C3 deficiency or high-dose CR2-Crry resulted in steatosis, severe hepatic injury and high mortality compared to the wild-type controls. However, mice treated with low-dose CR2-Crry had increased hepatic proliferative responses and significantly decreased damage compared with these responses in wild-type controls. A large quantity of $\mathrm{C} 3$ deposition was observed in wild-type mice at $48 \mathrm{~h}$ after partial hepatectomy, whilst C3 immunohistochemical tissue staining was absent in $\mathrm{C}^{-/-}$mice and mice treated with high-dose CR2-Crry. Furthermore, a low level of $\mathrm{C} 3$ immunohistochemical staining was present in mice treated with low-dose CR2-Crry. In reconstitution experiments, it was indicated that the $\mathrm{C} 3 \mathrm{a}$ degradation product C3adesArg (also known as acylation-stimulating protein) is important in the balance between inflammation/injury and regeneration. These results suggest a balance between complement-dependent injury and regeneration (56).

The aforementioned study also indicated that either $\mathrm{C} 3$ deficiency or treatment with CR2-Crry after IRI resulted in improved hepatocyte proliferation and accelerated recovery from injury as compared with the corresponding values in the wild-type controls, a result which demonstrated that complement is not critical to the recovery of hepatic IRI. Of note, recovery in the one-dose CR2-Crry treatment group is better than that in the $\mathrm{C} 3$ deficiency group; these results suggested that a low level of complement rather than complement deficiency is beneficial for the recovery of hepatic IRI injury. Although the precise mechanisms of the divergent effects of complement on liver regeneration between IRI and partial hepatectomy models remain elusive, preliminary data suggest that the differences are related to the amount of complement produced during these insults. Levels of C3a have been moderately increased (3- to 5-fold) after $70 \%$ partial hepatectomy but sharply increased (30- to 50-fold) after IRI. It was postulated that moderate increases in complement, which occur after $70 \%$ partial hepatectomy, promote liver regeneration via activation of regenerative signaling pathways, whereas much higher increases in the expression of complement components, as occurs after IRI injury, may be hepatotoxic and/or impair hepatocyte proliferation and regeneration. This concept was supported by experiments by our group in which animals were treated with various concentrations of CR2-Crry after $70 \%$ partial hepatectomy; low concentrations of CR2-Crry had hepatoprotective effects, whereas high concentrations induced significant cytotoxicity. Administration of low concentrations of CR2-Crry immediately after the onset of reperfusion downregulated inflammatory cytokines, decreased hepatic neutrophil infiltration, inhibited liver cell apoptosis and necrosis, and reduced liver injury and mortality in a mouse 
model of hepatic IRI. These data further support the concept of a balance between the extent of IRI and an ability to regenerate that is determined by the level of complement activation (56).

Several agents that inhibit all or part of the complement system have demonstrated a protective effect against liver IRI: i) Complement depletion with cobra venom factor $(8,62)$; ii) treatment with C5aR antagonist in rats after hepatic IRI (9); iii) intravenous soluble complement receptor 1 given prior to reperfusion after hepatic ischemia (63); and iv) inhibition of the classical pathway by pre-ischemic administration of C1 inhibitor (64). Therefore, there appear to be multiple potential pharmacologic strategies to protect against hepatic IRI by targeting the complement cascade. However, from the evidence of experimental models, the strategies of targeting C3 may provide the most effective complement inhibition during hepatic IRI, which does not only inhibit the formation of both $\mathrm{C} 3 \mathrm{a}$ and C5a anaphylatoxins, but also inhibits the formation of the MAC, which may directly result in hepatocyte lysis. However, given the beneficial role for complement, particularly its putative role in liver recovery and regeneration and the potential danger of systemic complement inhibition, therapeutic inhibition of complement must be performed to protect from hepatic IRI as much as possible while increasing liver regeneration.

Of note, when liver regeneration is the major concern as compared with hepatic IRI injury (such as in massive liver resection and small-for-size liver transplantation), selective inhibition at certain points in the complement pathway, rather than total complement inhibition (at the $\mathrm{C} 3$ point), may be more appropriate. Complement inhibition is considered a potential strategy for hepatic IRI, but upon complete inhibition at the C3 point, liver regeneration is decreased or may even stop completely. Zhou et al (33) demonstrated that the MAC is a potential target for the prevention of renal IRI. Overexpression of human CD55 and CD59 or treatment with human CD55 protects against renal IRI in mice (65). Zhang et al (66) reported that CD59-deficient mice had more severe liver dysfunction, as evidenced by increased aspartate aminotransferase levels and increased injury of liver parenchymal and nonparenchymal cells compared to CD59-sufficient mice during warm hepatic IR. Collectively, it may be hypothesized that the MAC has a predominant role in IRI but has a minor effect on liver regeneration, whereas more proximal activation products $(\mathrm{C} 3 \mathrm{a} / \mathrm{C} 5 \mathrm{a})$ are more important for liver regeneration. In a study by our group (11), CR2-CD59, a special complement inhibitor which may inhibit the formation of the MAC, was used to investigate how the complement works in the balance between liver injury and regeneration in a clinical setting of pharmacological inhibition. The results indicated that CR2-CD59 does not impair the generation of C3 and C5 activation products, but it may also ameliorate hepatic IRI, which is as effective as the C3 activation inhibitor CR2-Crry as reported previously (56).This result indicates that the MAC is crucial to hepatic IRI. Furthermore, unlike inhibition of C3 or C5, CR2-CD59 does not only protect against hepatic IRI but also significantly improves liver regeneration after partial hepatectomy, even when the hepatectomy is combined with IRI. Of note, CR2-CD59 may also improve long-term survival from 0 to $70 \%$ after $90 \%$ hepatectomy (11). This result demonstrates that specific inhibition of the MAC is an optimal potential therapeutic strategy to improve liver regeneration and reduce injury. In addition, inhibiting complement late in the pathway is less likely to disrupt normal immune homeostatic functions and host defense than earlier inhibition, which is an important consideration in the transplant recipient (11). These results suggest that the extent of complement activation may tip the balance between injury and the ability of the liver to regenerate (56).

\section{Future perspective}

Hepatic IRI is unavoidable in the process of liver operations. Data from animal studies indicate that complement is important in both hepatic IRI and recovery. At present, there is no effective pharmacological therapy for hepatic IRI in humans. Balanced approaches of complement inhibition or selective inhibition at certain points in the complement pathway (which may be obtained by temporal treatment of selective targeted inhibitors) are attractive strategies for reducing inflammation, minimizing hepatic IRI and enhancing liver recovery after hepatic IRI-inducing procedures, such as liver resection and transplantation. The present review shed new light on the role of the complement system in hepatic IRI and the failure of liver regeneration. Evidence from preclinical studies has revealed that regulation of the complement system represents a potential promising intervention strategy for patients undergoing massive liver resection or liver transplantation.

To date, studies on the complement system in hepatic IRI and liver regeneration have mainly focused on animal models of various liver diseases. A long road lies ahead before their clinical application. However, complement inhibitors such as eculizumab, Amy-101 and ravulizumab have entered or completed clinical trials (67-69). They have demonstrated good results in the treatment of diabetes mellitus, atypical hematologic urinary syndrome, paroxysmal nocturnal hemoglobinuria and Covid-19 (67-69). In conclusion, complement regulation strategies may be a promising application in the field of hepatic IRI and liver regeneration.

\section{Acknowledgements}

Not applicable.

\section{Funding}

The present study was supported in part by The National Natural Science Foundation of China (grant nos. 81771674 and 91949122), The 111 Project (grant no. D17011) and the Guangxi Key Research and Development Plan (grant no. 2018AD03001).

\section{Availability of data and materials}

Not applicable.

\section{Authors' contributions}

SQH, GDY, ZGH, YZ and CJL designed the study, wrote the manuscript, performed the literature research and selected the 
included studies. SQH and GDY provided critical intellectual revision. All authors read and approved the final manuscript.

\section{Ethics approval and consent to participate}

Not applicable.

\section{Patient consent for publication}

Not applicable.

\section{Competing interests}

The authors declare that they have no competing interests.

\section{References}

1. Gracia-Sancho J, Casillas-Ramirez A and Peralta C: Molecular pathways in protecting the liver from ischaemia/reperfusion injury: a 2015 update. Clin Sci (Lond) 129: 345-362, 2015.

2. Saidi RF and Kenari SK: Liver ischemia/reperfusion injury: an overview. J Invest Surg 27: 366-379, 2014.

3. Jaeschke H: Mechanisms of Liver Injury. II. Mechanisms of neutrophil-induced liver cell injury during hepatic ischemiareperfusion and other acute inflammatory conditions. Am J Physiol Gastrointest Liver Physiol 290: G1083-G1088, 2006.

4. Nastos C, Kalimeris K, Papoutsidakis N, Tasoulis MK Lykoudis PM, Theodoraki K, Nastou D, Smyrniotis V and Arkadopoulos N: Global consequences of liver ischemia/reperfusion injury. Oxid Med Cell Longev 2014: 906965, 2014

5. Thorgersen EB, Barratt-Due A, Haugaa H, Harboe M, Pischke SE, Nilsson PH and Mollnes TE: The role of complement in liver injury, regeneration, and transplantation. Hepatology 70 725-736, 2019.

6. 6. Zhou Y, Zhang J, Lei B, Liang W, Gong J, Zhao C, Yu J, Li X, Tang B and Yuan S: DADLE improves hepatic ischemia/reperfusion injury in mice via activation of the $\mathrm{Nrf} 2 / \mathrm{HO}-1$ pathway. Mol Med Rep 16: 6214-6221, 2017.

7. Lu L, Zhou H, Ni M, Wang X, Busuttil R, Kupiec-Weglinski J and Zhai Y: Innate immune regulations and liver ischemiareperfusion injury. Transplantation 100: 2601-2610, 2016.

8. Jaeschke H, Farhood A, Bautista AP, Spolarics Z and Spitzer JJ: Complement activates Kupffer cells and neutrophils during reperfusion after hepatic ischemia. Am J Physiol 264: G801-G809, 1993

9. Arumugam TV, Woodruff TM, Stocks SZ, Proctor LM, Pollitt S, Shiels IA, Reid RC, Fairlie DP and Taylor SM: Protective effect of a human C5a receptor antagonist against hepatic ischaemiareperfusion injury in rats. J Hepatol 40: 934-941, 2004.

10. Atkinson C, He S, Morris K, Qiao F, Casey S, Goddard M and Tomlinson S: Targeted complement inhibitors protect against posttransplant cardiac ischemia and reperfusion injury and reveal an important role for the alternative pathway of complement activation. J Immunol 185: 7007-7013, 2010.

11. Marshall KM, He S, Zhong Z, Atkinson C and Tomlinson S Dissecting the complement pathway in hepatic injury and regeneration with a novel protective strategy. J Exp Med 211: $1793-1805,2014$

12. Montalvo-Jave EE, Escalante-Tattersfield T, Ortega-Salgado JA, Piña E and Geller DA: Factors in the pathophysiology of the live ischemia-reperfusion injury. J Surg Res 147: 153-159, 2008.

13. Quesnelle KM, Bystrom PV and Toledo-Pereyra LH: Molecular responses to ischemia and reperfusion in the liver. Arch Toxicol 89: 651-657, 2015

14. Klune JR and Tsung A: Molecular biology of liver ischemia-reperfusion injury: Established mechanisms and recent advancements. Surg Clin North Am 90: 665-677, 2010.

15. Ye J, Xue M, Liu Y, Zhu S, Li Y, Liu X, Cai D, Rui J and Zhang L: Diosbulbin B-induced mitochondria-dependent apoptosis in L-02 hepatocytes is regulated by reactive oxygen speciesmediated autophagy. Front Pharmacol 10: 676, 2019.

16. Chavez-Cartaya R, Jamieson NV, Ramirez P, Marin J and Pino-Chavez G: Free radical scavengers to prevent reperfusion injury following warm liver ischemia. Transplant Proc 31: 2439-2440, 1999.
17. Cheng Y and Rong J: Therapeutic potential of heme oxygenase1-carbon monoxide system against ischemia-reperfusion injury. Curr Pharm Des 23: 3884-3898, 2017.

18. Li JY, Gu X, Zhang WH, Jia S and Zhou Y: GdCl3 abates hepatic ischemia-reperfusion injury by inhibiting apoptosis in rats. Hepatobiliary Pancreat Dis Int 8: 518-523, 2009.

19. Oliveira THC, Marques PE, Proost P and Teixeira MMM: Neutrophils: A cornerstone of liver ischemia and reperfusion injury. Lab Invest 98: 51-62, 2018.

20. Sun L, Wu Q, Nie Y, Cheng N, Wang R, Wang G, Zhang D, $\mathrm{He} \mathrm{H}$, Ye RD and Qian F: A role for MK2 in enhancing neutrophil-derived ROS production and aggravating liver ischemia-reperfusion injury. Front Immunol 9: 2610, 2018.

21. Jaeschke H, Farhood A and Smith CW: Neutrophils contribute to ischemia/reperfusion injury in rat liver in vivo. FASEB $\mathrm{J} 4$ : 3355-3359, 1990

22. Jaeschke H, Bautista AP, Spolarics Z and Spitzer JJ: Superoxide generation by Kupffer cells and priming of neutrophils during reperfusion after hepatic ischemia. Free Radic Res Commun 15: 277-284, 1991.

23. Woodcock SA, Kyriakides C, Wang Y, Austen WG Jr, Moore FD Jr, Valeri R, Hartwell D and Hechtman HB: Soluble P-selectin moderates complement dependent injury. Shock 14: 610-615, 2000

24. Pemberton M, Anderson G, Vetvicka V, Justus DE and Ross GD: Microvascular effects of complement blockade with soluble recombinant CR1 on ischemia/reperfusion injury of skeletal muscle. J Immunol 150: 5104-5113, 1993.

25. Panagiotou A, Trendelenburg M and Osthoff M: The lectin pathway of complement in myocardial ischemia/reperfusion injury-review of its significance and the potential impact of therapeutic interference by $\mathrm{C} 1$ esterase inhibitor. Front Immunol 9: 1151, 2018.

26. Arumugam TV, Magnus T, Woodruff TM, Proctor LM, Shiels IA and Taylor SM: Complement mediators in ischemia-reperfusion injury. Clin Chim Acta 374: 33-45, 2006.

27. Schieferdecker HL, Schlaf G, Jungermann K and Götze O Functions of anaphylatoxin C5a in rat liver: Direct and indirect actions on nonparenchymal and parenchymal cells. Int Immunopharmacol 1: 469-481, 2001.

28. Wiese AV, Ender F, Quell KM, Antoniou K, Vollbrandt T, Konig P, Kohl J and Laumonnier Y: The C5a/C5aR1 axis controls the development of experimental allergic asthma independent of LysM-expressing pulmonary immune cells. PLoS One 12: e0184956, 2017.

29. Guo RF and Ward PA: Role of C5a in inflammatory responses. Annu Rev Immunol 23: 821-852, 2005.

30. Winwood PJ and Arthur MJ: Kupffer cells: Their activation and role in animal models of liver injury and human liver disease. Semin Liver Dis 13: 50-59, 1993.

31. Zhang LY, Zhan DL, Chen YY, Wang WH, He CY, Lin Y, Lin YC and Lin ZN: Aflatoxin B1 enhances pyroptosis of hepatocytes and activation of Kupffer cells to promote liver inflammatory injury via dephosphorylation of cyclooxygenase-2: An in vitro, ex vivo and in vivo study. Arch Toxicol 93: 3305-3320, 2019.

32. Yu Y, Zhong J, Peng L, Wang B, Li S, Huang H, Deng Y, Zhang H, Yang R, Wang C, et al: Tacrolimus downregulates inflammation by regulating pro-/anti-inflammatory responses in LPS-induced keratitis. Mol Med Rep 16: 5855-5862, 2017.

33. Zhou W, Farrar CA, Abe K, Pratt JR, Marsh JE, Wang Y, Stahl GL and Sacks SH: Predominant role for C5b-9 in renal ischemia/reperfusion injury. J Clin Invest 105: 1363-1371, 2000.

34. Vollmar B, Menger MD, Glasz J, Leiderer R and Messmer K: Impact of leukocyte-endothelial cell interaction in hepatic ischemia-reperfusion injury. Am J Physiol 267: G786-G793, 1994.

35. Wang L, Vijayan V, Jang MS, Thorenz A, Greite R, Rong S, Chen R, Shushakova N, Tudorache I, Derlin K, et al: Labile heme aggravates renal inflammation and complement activation after ischemia reperfusion injury. Front Immunol 10: 2975, 2019.

36. Lai W, Xie X, Zhang X, Wang Y, Chu K, Brown J, Chen L and Hong G: Inhibition of complement drives increase in early growth response proteins and neuroprotection mediated by Salidroside after cerebral ischemia. Inflammation 41: 449-463, 2018.

37. Schönermark M, Deppisch R, Riedasch G, Rother K and Hänsch GM: Induction of mediator release from human glomerular mesangial cells by the terminal complement components C $5 \mathrm{~b}-9$. Int Arch Allergy Appl Immunol 96: 331-337, 1991.

38. Hattori R, Hamilton KK, McEver RP and Sims PJ: Complement proteins C5b-9 induce secretion of high molecular weight multimers of endothelial von Willebrand factor and translocation of granule membrane protein GMP-140 to the cell surface. J Biol Chem 264: 9053-9060, 1989. 
39. Fondevila C, Shen XD, Tsuchihashi S, Uchida Y, Freitas MC, Ke B, Busuttil RW and Kupiec-Weglinski JW: The membrane attack complex (C5b-9) in liver cold ischemia and reperfusion injury. Liver Transpl 14: 1133-1141, 2008.

40. He S, Atkinson C, Evans Z, Ellett JD, Southwood M, Elvington A, Chavin KD and Tomlinson S: A role for complement in the enhanced susceptibility of steatotic livers to ischemia and reperfusion injury. J Immunol183: 4764-4772, 2009.

41. Mao SA, Glorioso JM and Nyberg SL: Liver regeneration. Trans Res 163: 352-362, 2014.

42. Ibrahim S and Weiss TS: Augmenter of liver regeneration: Essential for growth and beyond. Cytokine Growth Factor Rev 45: 65-80, 2019.

43. Tao Y, Wang M, Chen E and Tang H: Liver regeneration. Mediators Inflamm 2017: 4256352, 2017.

44. Sergeeva O, Sviridov E and Zatsepin T: Noncoding RNA in liver regeneration-from molecular mechanisms to clinical implications. Semin Liver Dis 40: 70-83, 2019.

45. Szijártó A and Fülöp A: Triggered liver regeneration: From experimental model to clinical implications. European surgical research. Eur Surg Res 54: 148-161, 2015.

46. Mohammed FF and Khokha R: Thinking outside the cell Proteases regulate hepatocyte division. Trends Cell Biol 15: $555-563,2005$

47. Jia C: Advances in the regulation of liver regeneration. Expert Rev Gastroenterol Hepatol 5: 105-121, 2011.

48. Michalopoulos GK: Liver regeneration. J Cell Physiol 213 : 286-300, 2007.

49. Fausto $\mathbf{N}$ and Riehle KJ: Mechanisms of liver regeneration and their clinical implications. J Hepatobiliary Pancreat Surg 12 181-189, 2005

50. Strey CW, Markiewski M, Mastellos D, Tudoran R, Spruce LA, Greenbaum LE and Lambris JD: The proinflammatory mediators C3a and C5a are essential for liver regeneration. J Exp Med 198: 913-923, 2003

51. Markiewski MM, Mastellos D, Tudoran R, DeAngelis RA, Strey CW, Franchini S, Wetsel RA, Erdei A and Lambris JD: $\mathrm{C} 3 \mathrm{a}$ and $\mathrm{C} 3 \mathrm{~b}$ activation products of the third component of complement (C3) are critical for normal liver recovery after toxic injury. J Immunol 173: 747-754, 2004.

52. Mastellos D, Papadimitriou JC, Franchini S, Tsonis PA and Lambris JD: A novel role of complement: Mice deficient in the fifth component of complement (C5) exhibit impaired liver regeneration. J Immunol 166: 2479-2486, 2001

53. Clark A, Weymann A, Hartman E, Turmelle Y, Carroll M, Thurman JM, Holers VM, Hourcade DE and Rudnick DA: Evidence for non-traditional activation of complement factor C3 during murine liver regeneration. Mol Immunol 45: 3125-3132, 2008.

54. Daveau M, Benard M, Scotte M, Schouft MT, Hiron M, Francois A, Salier JP and Fontaine M: Expression of a functional C5a receptor in regenerating hepatocytes and its involvement in a proliferative signaling pathway in rat. J Immunol 173: 3418-3424, 2004.

55. Castellano G, Franzin R, Sallustio F, Stasi A, Banelli B, Romani M, De Palma G, Lucarelli G, Divella C, Battaglia M, et al: Complement component C5a induces aberrant epigenetic modifications in renal tubular epithelial cells accelerating senescence by Wnt $4 / \beta$ catenin signaling after ischemia/reperfusion injury. Aging (Albany NY) 11: 4382-4406, 2019.

56. He S, Atkinson C, Qiao F, Cianflone K, Chen X and Tomlinson S: A complement-dependent balance between hepatic ischemia/ reperfusion injury and liver regeneration in mice. J Clin Invest 119: 2304-2316, 2009.
57. Song H, He C, Knaak C, Guthridge JM, Holers VM and Tomlinson S: Complement receptor 2-mediated targeting of complement inhibitors to sites of complement activation. J Clin Invest 111: 1875-1885, 2003.

58. Alawieh A, Langley EF, Weber S, Adkins D and Tomlinson S Identifying the role of complement in triggering neuroinflammation after traumatic brain injury. J Neurosci 38: 2519-2532, 2018.

59. Schnabolk G, Parsons N, Obert E, Annamalai B, Nasarre C, Tomlinson S, Lewin AS and Rohrer B: Delivery of CR2-fH using AAV vector therapy as treatment strategy in the mouse model of choroidal neovascularization. Mol Ther Methods Clin Dev 9: 1-11, 2017.

60. Parsons N, Annamalai B, Obert E, Schnabolk G, Tomlinson S and Rohrer B: Inhibition of the alternative complement pathway accelerates repair processes in the murine model of choroidal neovascularization. Mol Immunol 108: 8-12, 2019.

61. Banda N, Levitt B, Glogowska M, Thurman J, Takahashi K, Stahl G, Tomlinson S, Arend W and Holers V: Targeted inhibition of the complement alternative pathway with complement receptor 2 and factor $\mathrm{H}$ attenuates collagen antibody-induced arthritis in mice. J Immunol 183: 5928-5937, 2009.

62. Wang B, Xu H, Li J, Gao HM, Xing YH, Lin Z, Li HJ, Wang YQ and Cao SH: Complement depletion with cobra venom factor alleviates acute hepatic injury induced by ischemia-reperfusion. Mol Med Rep 18: 4523-4529, 2018.

63. Lehmann TG, Koeppel TA, Münch S, Heger M, Kirschfink M, Klar E and Post S: Impact of inhibition of complement by sCR1 on hepatic microcirculation after warm ischemia. Microvasc Res 62: 284-292, 2001.

64. Heijnen BH, Straatsburg IH, Padilla ND, Van Mierlo GJ, Hack CE and Van Gulik TM: Inhibition of classical complement activation attenuates liver ischaemia and reperfusion injury in a rat model. Clin Exp Immunol 143: 15-23, 2006.

65. Bongoni A, Lu B, Salvaris E, Roberts V, Fang D, McRae J, Fisicaro N, Dwyer K and Cowan P: Overexpression of human CD55 and CD59 or treatment with human CD55 protects against renal ischemia-reperfusion injury in mice. J Immunol 198: 4837-4845, 2017

66. Zhang J, Hu W, Xing W, You T, Xu J, Qin X and Peng Z: The protective role of CD59 and pathogenic role of complement in hepatic ischemia and reperfusion injury. Am J Pathol 179: 2876-2884, 2011

67. Mastaglio S, Ruggeri A, Risitano AM, Angelillo P, Yancopoulou D, Mastellos DC, Huber-Lang M, Piemontese S, Assanelli A, Garlanda C, et al: The first case of COVID-19 treated with the complement C3 inhibitor AMY-101. Clin Immunol 215: 108450, 2020.

68. Wang Y, Johnston K, Popoff E, Myren KJ, Cheung A, Faria C and Tomazos I: A US cost-minimization model comparing ravulizumab versus eculizumab for the treatment of atypical hemolytic uremic syndrome. J Med Econ 10: 1-13, 2020.

69. Peipert JD, Kulasekararaj AG, Gaya A, Langemeijer SMC, Yount S, Gonzalez-Fernandez FA, Ojeda Gutierrez E, Martens C, Sparling A, Webster KA, et al: Patient preferences and quality of life implications of ravulizumab (every 8 weeks) and eculizumab (every 2 weeks) for the treatment of paroxysmal nocturnal hemoglobinuria. PLoS One 15: e0237497, 2020.

This work is licensed under a Creative Commons Attribution-NonCommercial-NoDerivatives 4.0 International (CC BY-NC-ND 4.0) License. 